\title{
Biomechanik des Ellenbogengelenks
}

\author{
T. Leschinger, K. Wegmann, M. Hackl, L. P. Müller \\ Schwerpunkt für Unfall-, Hand- und Ellenbogenchirurgie, Universitätsklinik zu Köln
}

In seiner Gesamtheit hat das Ellenbogengelenk für das Heran- und Wegführen der Hand sowie deren stabiler Positionierung im Raum eine elementare Bedeutung. Aus diesem Grunde ist neben einer intakten Mobilität eine adäquate Sta- bilität unverzichtbar. Für diese Aufgaben sind die biomechanische Integrität des Gelenks und das Verständnis vom Zusammenspiel der Strukturen am Ellenbogen unerlässlich.

\section{Einleitung}

Der Ellenbogen bildet als gelenkige Verbindung zwischen Ober- und Unterarm das biomechanisch bedeutsame Element für die Funktionalität der oberen Extremität. Ein Grund hierfür ist das komplexe Zusammenspiel der Teilgelenke des Ellenbogens, die aus dem proximalen Gelenk von Radius und Ulna und den Artikulationen von Humerus und Ulna sowie Humerus und Radius bestehen.

Eine weitere Ursache ist, dass der Ellenbogen zwischen Ober- und Unterarm positioniert ist und somit durch den langen Hebelarm bei Krafteinleitung an den Händen großen Kräften ausgesetzt wird. Berechnet man die am Ellenbogen wirkenden Kräfte und ihre Effekte auf die kleinen Knochen bzw. kraftübertragenden Gelenkflächen, muss man feststellen, dass die Strukturen des Ellenbogengelenks in ähnlicher Höhe beansprucht werden wie die der unteren Extremität. Während beim Anziehen Kompressionskräfte im Ellenbogengelenk von ca. $300 \mathrm{~N}$ berichtet werden, können schon beim Abstützen des Armes Gelenkreaktionskräfte von $1700 \mathrm{~N}$ auf dem medialen und $800 \mathrm{~N}$ auf dem lateralen Kompartiment beobachtet werden. Folglich kann der Ellenbogen nachhaltig als lasttragendes Gelenk eingestuft werden und ist entsprechend zu werten.

\section{Anatomie der Skelettanteile}

Das Ellenbogengelenk wird knöchern von dem distalen Humerus, dem Radius und der Ulna gebildet, die von einer gemeinsamen Kapsel umhüllt werden (Abb. 1).

\section{Distaler Humerus}

Der distale Anteil des Humerus besteht aus zwei Kondylen:

- der Trochlea humeri als Gelenkfläche für die Ulna und

- dem Capitulum humeri als Artikulationsfläche des Radius.

\section{Abkürzungen}

ALCL akzessorisches laterales Kollateralband

A-MCL anteriores Bündel des medialen Kollateralbandes

DRUG distales Radioulnargelenk

$\mathrm{LCL} \quad$ laterales Kollateralband

LUCL laterales ulnares Kollateralband

$\mathrm{MCL} \quad$ mediales Kollateralband

PLRI posterolaterale Rotationsinstabilität

P-MCL posteriores Bündel des medialen Kollateralbandes

PRUG proximales Radioulnargelenk

$\mathrm{RCL} \quad$ radiales Kollateralband 


\section{Grundlagen}

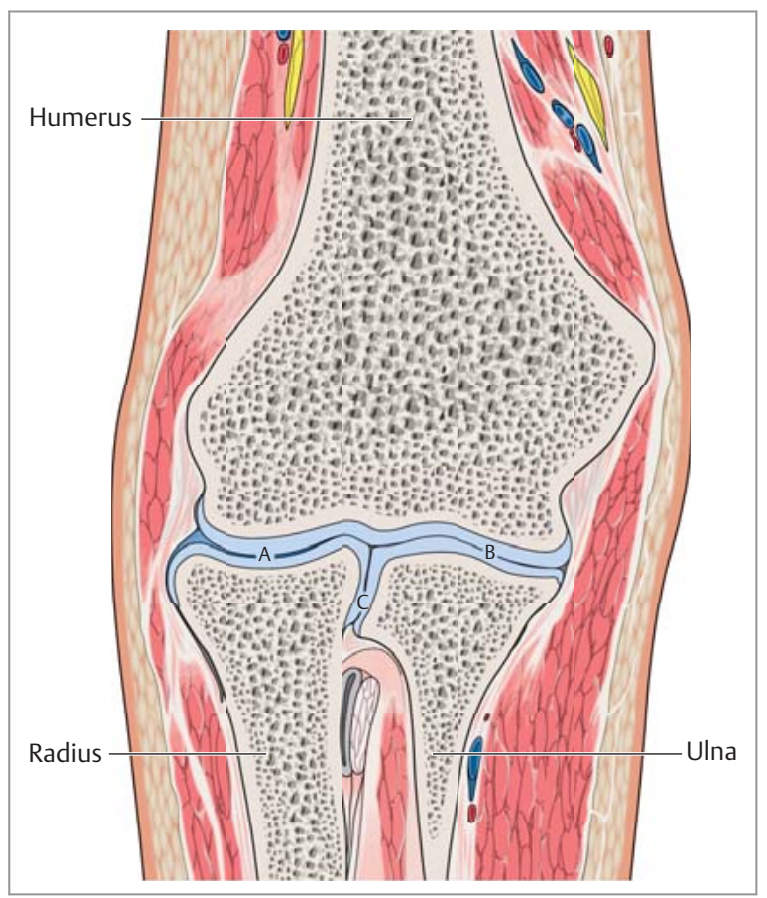

Abb. 1 - Die 3 Teilgelenke des Ellenbogens: $A$ = radiokapitellares Gelenk, B = ulnohumerales Gelenk, C = proximales radioulnares Gelenk [1].

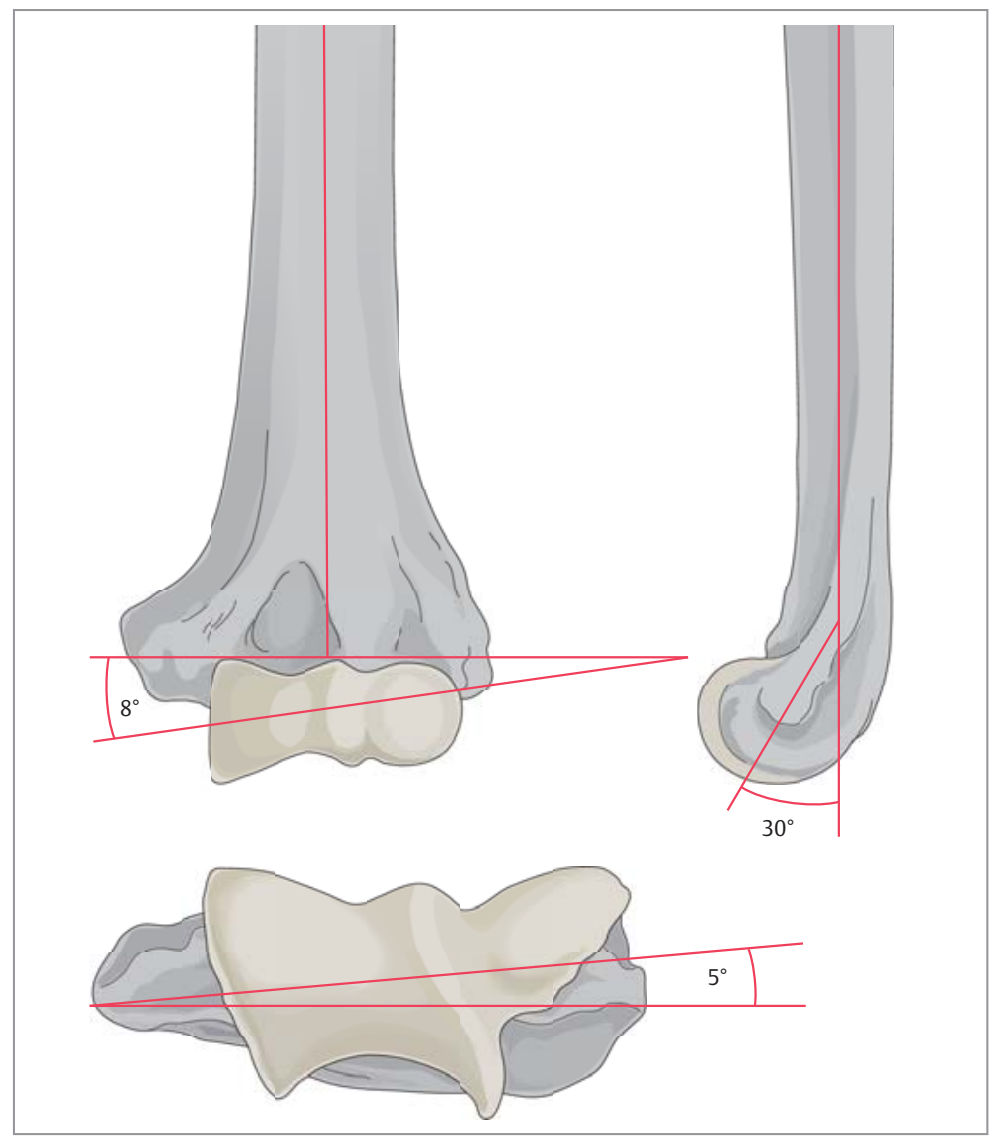

Abb. 2 - Die Achsen des distalen Humerus. Er zeigt sich in der Sagittalebene um $30^{\circ}$ nach ventral gekippt, in der Transversalebene um ca. $5^{\circ}$ einwärts rotiert und in der Frontalebene $6-8^{\circ}$ geneigt [1].
Der distale Anteil des Humerus ist in der Sagittalebene um $30^{\circ}$ nach anterior gekippt, in der Transversalebene um ca. $5^{\circ}$ einwärts rotiert und in der Frontalebene $6-8^{\circ}$ geneigt (Abb. 2) [1]. Proximal lateral ermöglicht die Crista supracondylaris lateralis den Ansatz des M. brachioradialis und des M. extensor carpi radialis sowie dorsalseitig des M. triceps brachii. Aus ihrer Verbreiterung geht der Epicondylus lateralis (radialis) hervor, an dem die lateralen (radialen) Kollateralbänder und die Supinator-Extensoren-Muskelgruppe ansetzen. Medial hat der Epicondylus medialis (ulnaris) als Ansatz der medialen (ulnaren) Kollateralbänder sowie der Flexorund Pronatorenmuskulatur diese Funktion inne. An seiner Dorsalseite verläuft der N. ulnaris im Sulcus nervi ulnaris.

Anterior am distalen Humerus befinden sich auf der medialen Seite die Fossa coronoidea und lateral die Fossa radialis, die in Flexion als Gleitlager für den Radiuskopf bzw. den Processus coronoideus dienen. Als posteriores Äquivalent ermöglicht die Fossa olecrani der Spitze des Olekranons der Ulna die Beweglichkeit in Extension (Abb. 3).

\section{Prinzipien}

\section{Chirurgische Relevanz}

Osteophytäre Anbauten bzw. freie Gelenkkörper können durch Verlegung der Fossae zu einer eingeschränkten Beweglichkeit führen.

\section{Proximaler Radius}

Der proximale Radius besteht aus dem Caput radii (Radiuskopf), das im Durchschnitt eine Tiefe von 2,4 mm und einen Durchmesser von ca. $24 \mathrm{~mm}$ aufweist, an das sich distal das Collum radii (Radiushals) anschließt, das zu der Längsachse des Radiusschaftes einen Winkel von $15^{\circ}$ bildet $[1,2]$. Der Radiuskopf weist interindividuell große Unterschiede in seiner Form auf, ist jedoch in der Regel eher ellipsoid als kreisrund. Die Tuberositas radii am distalen Ende des Collum radii bildet die Ansatzfläche der Sehne des M. biceps brachii (Abb. 3). 


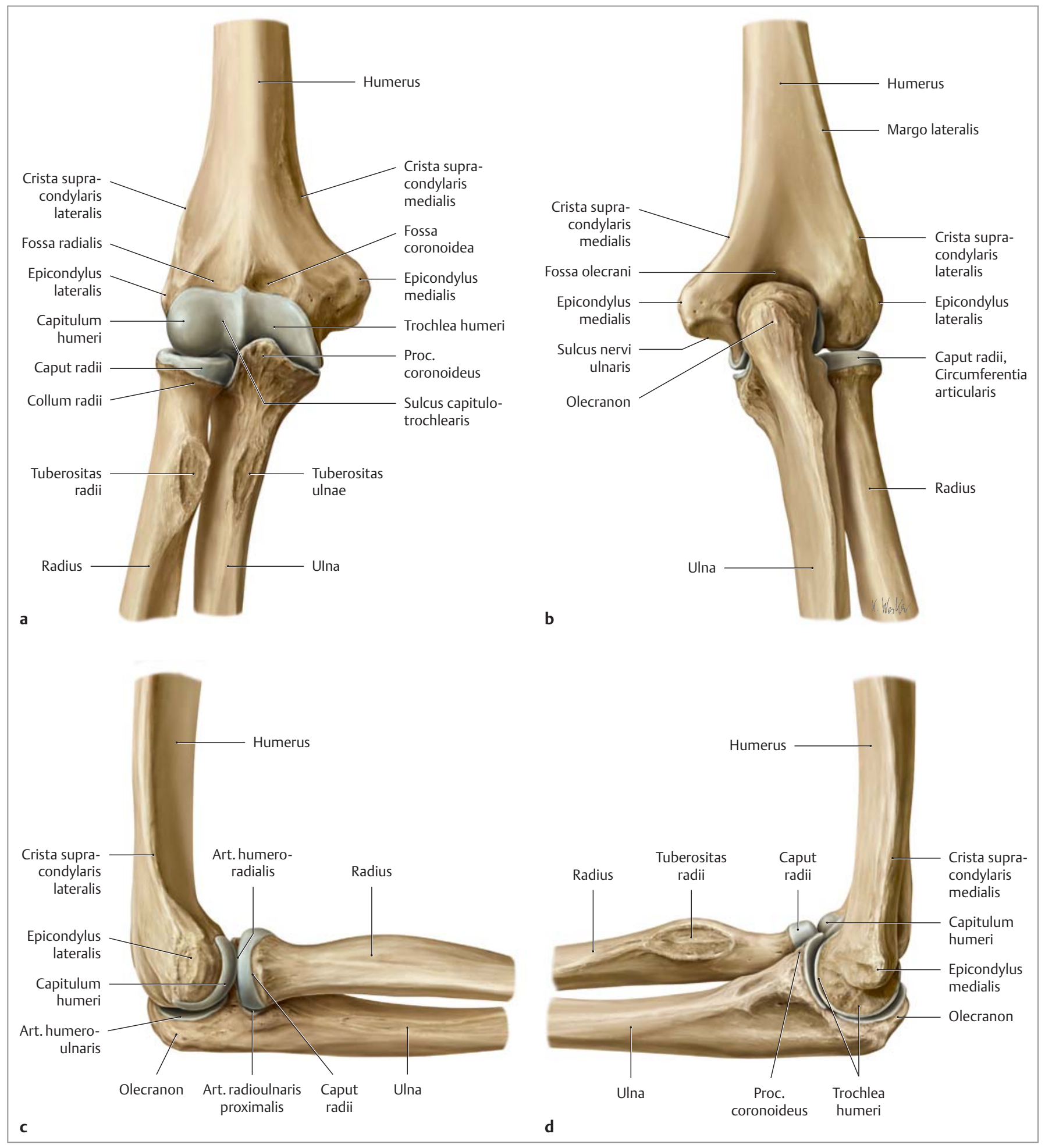

Abb. 3 - Artikulierende Skelettelemente eines rechten Ellenbogens [3]. a Ansicht von ventral. b Ansicht von dorsal. c Ansicht von lateral. d Ansicht von medial. 


\section{Grundlagen}

\section{Proximale Ulna}

Die proximale Ulna bildet durch den Processus coronoideus anterior und durch das Olekranon posterior - das als Ansatz für den M. triceps brachii dient - eine Art Zange um den distalen Humerus (Abb.4).

Medial des Processus coronoideus liegt die Incisura semilunaris ulnae, die bei den meisten Menschen eine knorpelfreie Zone aufweist, welche die Gelenkfläche der Ulna in einen anterioren und einen posterioren Abschnitt unterteilt. Diese knorpelfreie Zone weist eine durchschnittliche Breite von $5 \mathrm{~mm}$ auf und resultiert aus den Kontaktflächen und der Spannungsverteilung am Olekranon, da bei Extension/Flexion der Großteil der Kräfte über den posterioren und anterioren Anteil der ellipsoiden Incisura läuft (Abb. 5).

Sie ist in der Sagittalebene zur Ulnaschaftachse um ca. $30-45^{\circ}$ nach posterior geöffnet und korrespondiert hierbei zu der Trochlea des distalen Humerus (s.o.) (Abb. 6).

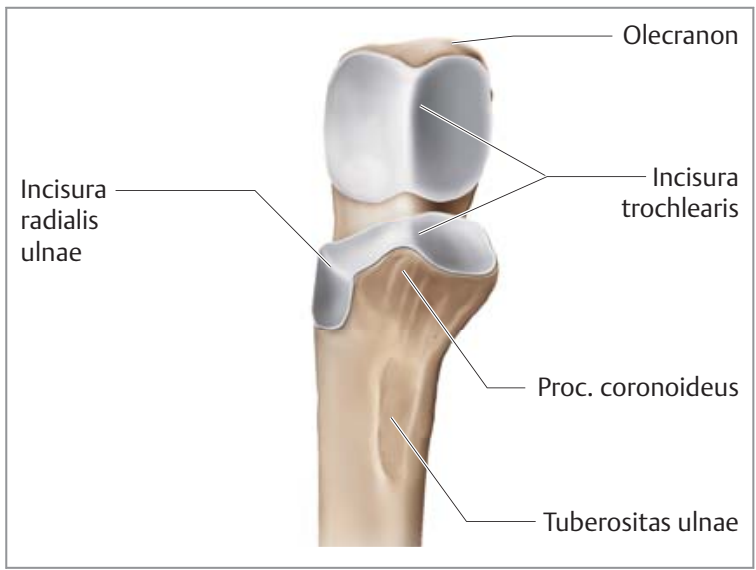

Abb. 4 - Proximale Ulna, Ansicht von ventral [4].

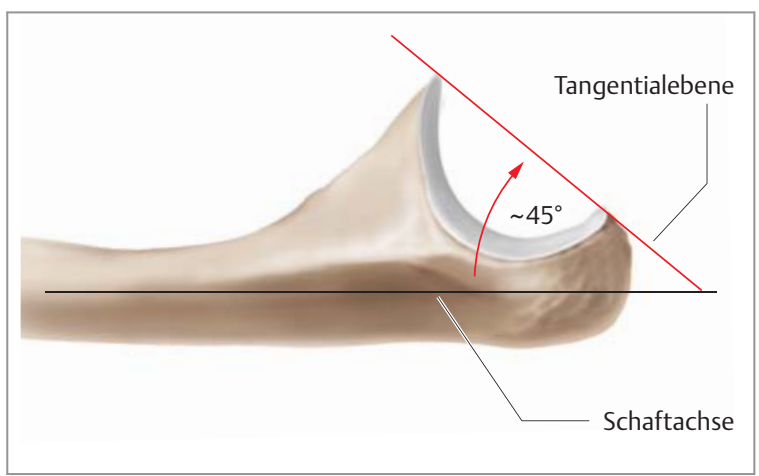

Abb. 6 - Ausrichtung der Incisura trochlearis [4].
Lateral des Koronoids liegt die Incisura radialis - als Artikulationsfläche für den Radiuskopf - und läuft in die Crista supinatoris aus, die den Ursprung des M. supinator und den Ansatz der dorsalen Anteile des lateralen Kollateralbandkomplexes bildet. Wie der Radiuskopf zeigt auch die proximale Ulna interindividuelle Unterschiede (Abb. 3).

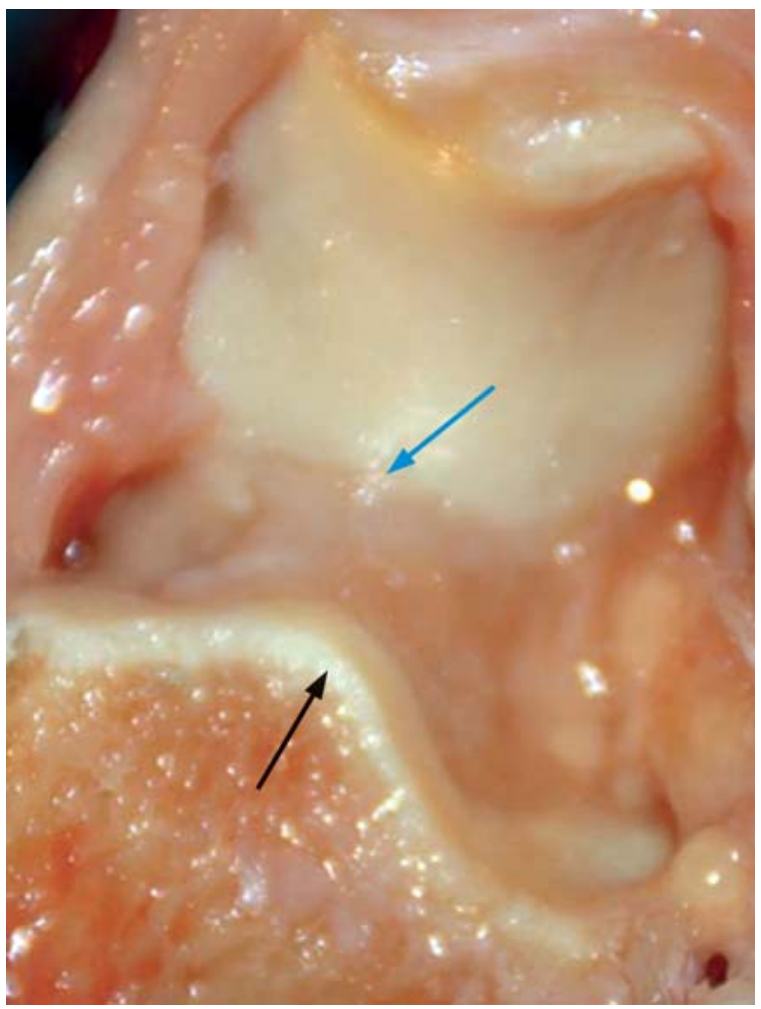

Abb. 5 - Knorpelfreie Zone am Leichenpräparat (schwarzer Pfeil = Processus coronoideus, blauer Pfeil = Übergang des posterioren knorpeligen Anteils des Olekranons in die knorpelfreie Zone) [1].

\section{Prinzipien}

\section{Chirurgische Relevanz}

Anatomische Platten können bei der Versorgung von Frakturen der proximalen Ulna aufgrund von morphologischen und geschlechtsspezifischen Unterschieden häufig keine optimale Lösung bieten. Besonders im Rahmen von Monteggia-Frakturen, die mit einer Instabilität des radiohumeralen Gelenks einhergehen, besteht die Gefahr, bei der operativen Versorgung die anteriore Angulation der Ulna durch die vorgefertigte Platte nicht optimal einzustellen. Dies kann eine Subluxationsstellung des Radiuskopfes aus dem radiohumeralen Gelenk zur Folge haben. 
Blickt man von dorsal auf das extendierte Ellenbogengelenk, liegen das Olekranon und die Epikondylen auf einer geraden Linie, wohingegen sie bei $90^{\circ}$ flektiertem Ellenbogen ein inverses gleichschenkeliges Dreieck bilden.

\section{Prinzipien}

\section{Chirurgische Relevanz}

Bei dislozierten Frakturen oder Luxationen kommt es zu einer Aufhebung dieser Anordnung.

\section{Die Teilgelenke des Ellenbogens}

\section{Humeroradialgelenk}

Als Humeroradialgelenk artikuliert lateral das konvexe Kapitulum des distalen Humerus mit dem konkaven Radiuskopf. Das Kapitulum ist anterior mit einer ca. $2 \mathrm{~mm}$ dicken Knorpelschicht überzogen, sodass posterior in der knorpelfreien Zone das Anbringen von Osteosynthesematerial möglich ist [6].

Biomechanisch stellt das Humeroradialgelenk ein Kugelgelenk mit 3 Freiheitsgraden dar, ist jedoch gleichzeitig ein wichtiger translatorischer Stabilisator. Es generiert Widerstand gegen Valgusstress am Ellenbogen und verhindert die posteriore Dislokation bei Beugewinkeln von mehr als $90^{\circ}$.

Die Intaktheit des Humeroradialgelenks ist wichtig für die translatorische Stabilität des Ellenbogengelenks.

\section{Prinzipien}

\section{Chirurgische Relevanz}

Aufgrund des konkaven Radiuskopfes sollte das Einbringen von Schrauben unter Sicht erfolgen, da die radiologische Beurteilung schwierig ist.

\section{Humeroulnargelenk}

Auf der medialen Seite artikuliert die Trochlea, die eine hyperbolische Oberfläche aufweist, mit der Incisura semilunaris ulnae und bildet das Humeroulnargelenk. Die Trochlea ist über einen Bogen von ca. $300^{\circ}$ mit hyalinem Knorpel überzogen. Das Humeroulnargelenk stellt ein modifiziertes, idealisiertes Scharniergelenk (s.u.) mit einem Freiheitsgrad dar und gestattet den größten Anteil der knöchernen Gelenkführung [6]. Der Processus coronoideus stellt dabei den wichtigsten Block gegen die posteriore Verschiebung bei Ellenbogenflexion dar.

Das Humeroulnargelenk ist das wichtigste stabilisierende Teilgelenk.

\section{Prinzipien}

\section{Chirurgische Relevanz}

Bei der anatomischen Rekonstruktion von gelenktragenden Frakturen der proximalen Ulna muss auf das Realignment der dorsalen Kortikalis geachtet werden, um die Aufnahmefläche für die Trochlea nicht zu verringern.

Führt man eine Olekranonosteotomie durch, empfiehlt es sich, den Sägeschnitt in dem knorpelfreien Bereich anzubringen.

\section{Proximales Radioulnargelenk}

Als drittes Teilgelenk artikulieren die Incisura radialis ulnae und die Zirkumferenz des Radiuskopfes im proximalen Radioulnargelenk (PRUG). Das anterolaterale Drittel der Circumferentia articularis ist nicht mit hyalinem Knorpel bedeckt und daher bei fehlender subchondraler Lamelle vermehrt frakturgefährdet.

Das PRUG wird durch das Lig. anulare stabilisiert. Es stellt sich als Radgelenk oder besser als Zapfengelenk mit zylindrischen Gelenkflächen und einem Freiheitsgrad dar [6].

\section{Prinzipien}

\section{Chirurgische Relevanz}

Wichtig beim Einbringen des Osteosynthesematerials ist die Beachtung der Safe-Zone am Radiuskopf (ca. $133^{\circ}$ des Umfangs des Radiuskopfes, der nicht an der Artikulation mit der Incisura radialis der proximalen Ulna beteiligt ist), die als Bereich dienen sollte, in dem die Versorgung ohne biomechanische Probleme erfolgen kann (Abb. 7). 


\section{Grundlagen}

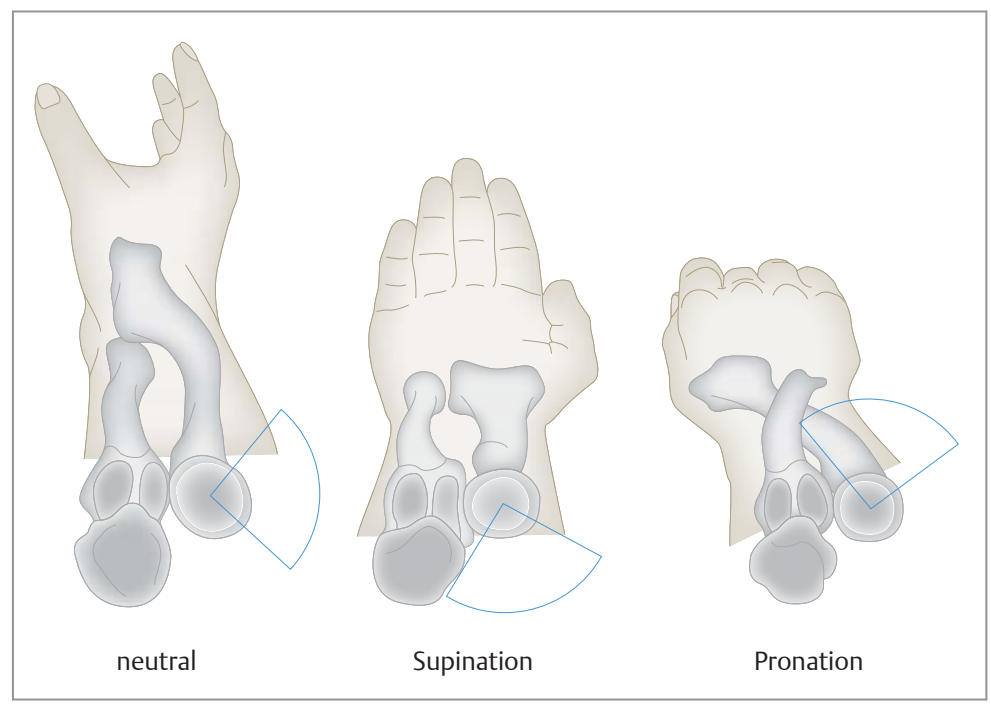

Abb. 7 - Safe-Zone am Radiuskopf streng lateral bei Lagerung des Armes in Neutralposition (links). Osteosynthesematerial, das in der Safe-Zone eingebracht wird, kommt bei Pro- und Supination nicht in Konflikt mit dem proximalen radioulnaren Gelenk [1].

Tabelle 1

Die 3 Teilgelenke des Ellenbogens.

\begin{tabular}{|ll}
\hline Gelenk & Gelenktyp \\
\hline Humeroradialgelenk & Kugelgelenk \\
\hline Humeroulnargelenk & Scharniergelenk \\
\hline proximales Radioulnargelenk & Radgelenk \\
& Zapfengelenk \\
& trochoidales Gelenk \\
\hline
\end{tabular}

Die Gelenktypen der 3 Teilgelenke des Ellenbogens fasst Tab. 1 zusammen.

\section{Der Unterarm als funktionelle Einheit}

Weist das Ellenbogengelenk im engeren Sinn nur die beschriebenen Teilgelenke auf, so muss es im funktionellen Sinne durch das distale Radioulnargelenk (DRUG) komplettiert werden. Dies ist wichtig, da die einzelnen Teilgelenke des Unterarms stets zusammen agieren und auch nur als intaktes System optimal die axiale Kraft entfalten und weiterleiten können.

Die Intaktheit der Membrana interossea als Verbindung zwischen Radius und Ulna ist hierbei wichtig. Die Fasern verlaufen schräg abwärts vom Radius zur Ulna. Dieses Element schützt vor Separation oder Migration von Radius und Ulna und trägt damit auch zum Transfer von Axialkräften vom Radius zur Ulna bei.

Werden am distalen Unterarm 80\% der aufgenommenen Kräfte über den Radius und nur 20\% über die Ulna geleitet, so erfolgt durch die Membran eine Krafttransmission von bis zu 50\% vom Radius zur Ulna. Hierdurch werden am Ellenbogen 50-60\% über die radiale Säule (Radiuskopf - Capitulum humeri) bzw. 40-50\% über die ulnare Säule (Processus coronoideus - Trochlea) auf den distalen Humerus übermittelt. Die in anatomischen Studien berichtete Festigkeitszunahme der Ulna und Festigkeitsabnahme des Radius von distal nach proximal wird hierdurch schlüssig.

Besonders bei Stürzen auf den ausgestreckten Arm kann dieses System jedoch wie eine rigide Säule wirken. Durch die axiale Fortleitung der Kraft kann es zu einem hohen Valgusmoment am Unterarm kommen, was zur Summation der Kraft im Radiuskopf und zu einer erheblichen Zugspannung am medialen Kollateralband (MCL) führen kann. Dies hat zur Folge, dass die Kraft im Radiuskopf aufgrund der Zugkraft am MCL größer wird als die extern applizierte Kraft, wohingegen die Belastung des Processus coronoideus deutlich abnimmt. Hierdurch kommt es zum Bruch des Radiuskopfes und zur Läsion des MCL.

\section{Prinzipien}

\section{Chirurgische Relevanz}

Eine weitere Relevanz hat die beschriebene Verbindung durch die oftmals übersehene Essex-Lopresti-Verletzung. Sie beschreibt eine Läsion des Radiuskopfes, der Membrana interossea und des distalen Radioulnargelenks (DRUG), die zu einer Proximalisierung des Radius führt. Dies kann in einem chronischen Fall der Essex-Lopresti-Verletzung zu einem radiokapitellaren Impingement und sekundär zu einem ulnokarpalen Impingement führen.

Klinisch muss hierbei neben Schmerzen über dem Unterarm auf ein schmerzhaftes bzw. hypermobiles DRUG geachtet werden. 


\section{Gelenkkapsel}

Die 3 Teilgelenke des Ellenbogens liegen in einer gemeinsamen, fibrösen Gelenkkapsel, die sich anterior von proximal der Fossa coronoidea und der Fossa radialis nach distal an den vorderen Rand des Processus coronoideus sowie an das Lig. anulare spannt. Posterior erstreckt sich die Kapsel von proximal der Fossa olecrani nach distal zum Lig. anulare und dem Rand des Olekranons. In Extension kommt es zur Anspannung der anterioren Kapselfasern, wodurch diese eine stabilisierende Funktion gegen Varus- und Valgusbelastungen übernehmen.

\section{Prinzipien}

\section{Chirurgische Relevanz}

Bei einem intraartikulären Erguss bzw. Hämarthros halten die Patienten das Ellenbogengelenk meist in $70-80^{\circ}$ Flexion, da so die schmerzärmste Position (aktuelle Ruhestellung) erreicht wird. Radiologisch kommt es durch den Hämarthros zum sogenannten Fat Pad Sign, d. i. ein Aufhellungsband an der Kapsel, das durch ihre Abhebung vom Knochen entsteht.
- Je nach Gelenkstellung variieren auch das Füllungsvolumen und die Spannung der Kapsel. Das größte Füllungsvolumen bzw. die geringste Kapselspannung

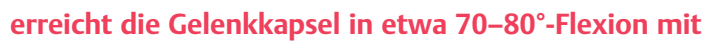
ca. $25-30 \mathrm{ml}$.

\section{Freiheitsgrade, Drehzentrum und Valguswinkel}

Das Ellenbogengelenk erlaubt im Wesentlichen Bewegungen in 2 Freiheitsgraden.

Das humeroulnare und humeroradiale Gelenk gestatten gemeinsam die Flexion und Extension und machen den Ellenbogen vereinfacht zu einem uniaxialen Scharniergelenk, dessen humeroulnare Gelenkachse mit zunehmender Beugestellung nach ventral wandert.

Die Bewegung der Pronation und Supination erfolgt im proximalen Radioulnargelenk (PRUG) in Kombination mit dem distalen Radioulnargelenk (DRUG) entlang der Drehachse des Unterarms. Das PRUG ist somit als trochoidales Gelenk zu klassifizieren.

Damit stellt der Ellenbogen biomechanisch ein zusammengesetztes, trochoidales Scharniergelenk dar (Abb. 8).

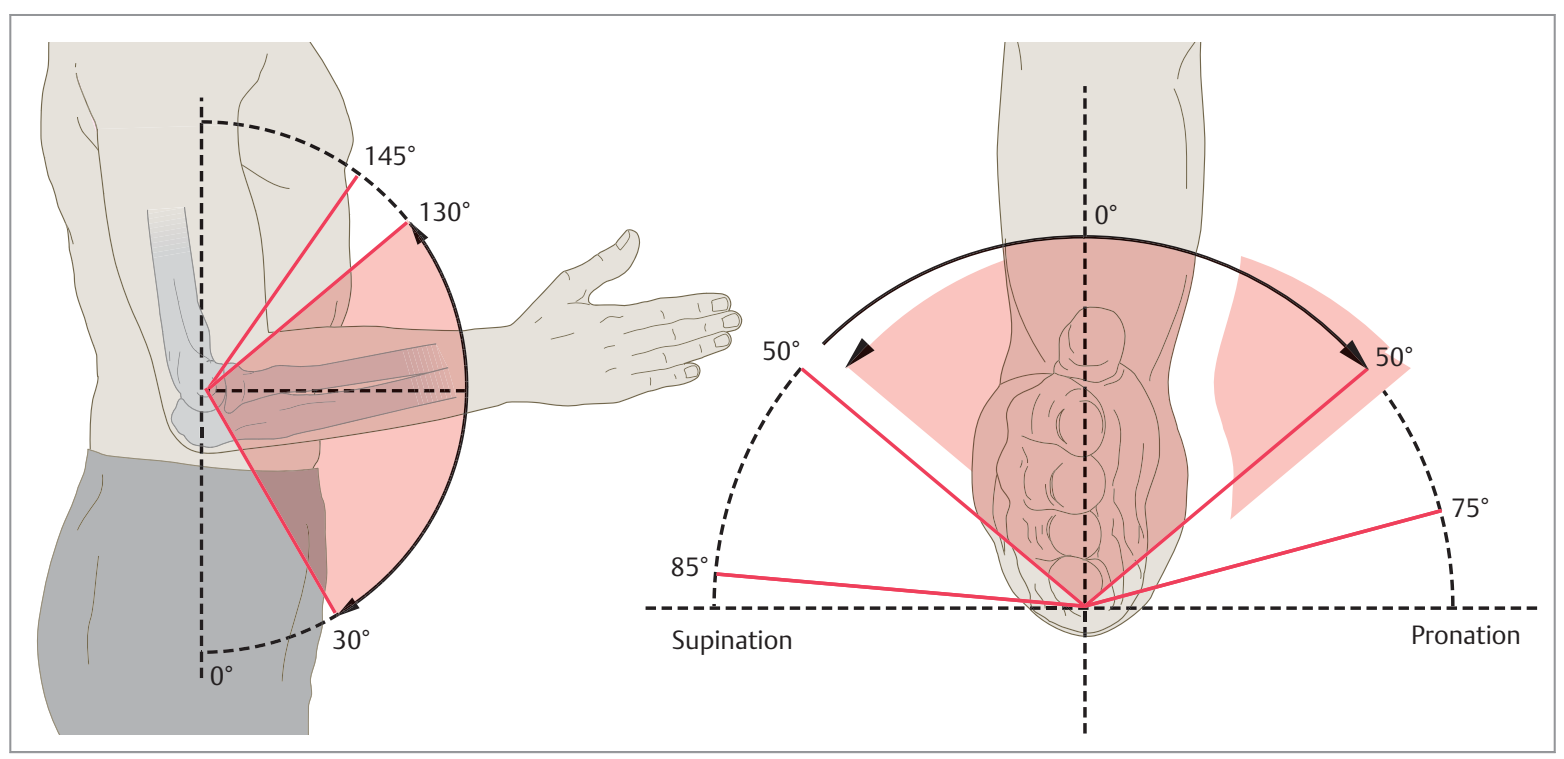

Abb. 8 - Das physiologische Bewegungsausmaß der Extension/Flexion liegt bei 0/0/145 (links). Die Pronation/Supination beträgt physiologischerweise $75 / 0 / 85$ (rechts); [1]. 


\section{Flexion und Extension}

Eine Extensions-Flexions-Bewegung des Ellenbogens wird durch das humeroulnare und das humeroradiale Gelenk erreicht. Das Drehzentrum für diese Bewegung befindet sich auf einer Fläche von etwa $2-3 \mathrm{~mm}$ Durchmesser an der Trochlea. Die individuellen Unterschiede des Drehzentrums sind gering, sodass die Drehachse vereinfacht dargestellt vom inferioren Anteil des medialen Epikondylus zum Zentrum des lateralen Kondylus zieht $[6,8]$.

Das maximal mögliche, physiologische Bewegungsausmaß dieser Bewegungsrichtung kann schon über die geometrischen Charakteristika der beteiligten Knochenstrukturen und den Anteil der knorpeligen Überdeckung der Gelenkfläche abgeschätzt werden. Der Winkelbereich der Gelenkfläche der Trochlea des Humerus beträgt $330^{\circ}$, während derjenige der Incisura trochlearis der Ulna ca. $190^{\circ}$ ausmacht. Die Differenz von entsprechend $140^{\circ}$ bestimmt somit das Bewegungsausmaß des Ellenbogens für die Flexion-Extension. Gleichzeitig finden sich $140^{\circ}$ Differenz zwischen dem Kreisabschnitt der Gelenkfläche des Capitulum humeri $\left(180^{\circ}\right)$ und derjenigen des proximalen Radiuskopfes $\left(40^{\circ}\right)$.

Die Bewegung selbst stellt prinzipiell eine „Gleitbewegung“ dar, die in den endgradigen $5-10^{\circ}$ in eine „Rollbewegung“ übergeht [1]. Grund für diese Rollbewegung und gleichzeitig Hauptlimitation des Bewegungsausmaßes sind die Knochenstrukturen, nämlich in Flexion der Kontakt des Processus coronoideus mit dem Boden der Fossa coronoidea und in Extension der Kontakt des Olekranons mit dem Boden der Fossa olecrani.

Als Weichteilhemmung ist zusätzlich eine Limitierung der Bewegung durch die Gelenkkapsel und den M. triceps brachii gegeben, die vor allem in Flexion auftritt. Der Bewegungsumfang steigt somit bei entspannter Muskulatur.

Die passive Ellenbogenflexion wird im Bewegungsumfang mit bis zu $146^{\circ}$ beschrieben, wohingegen der aktive Bewegungsumfang mit $0-142^{\circ}$ beziffert wird.

Das Bewegungsausmaß nimmt im Alter ab, während Kinder im Mittel noch eine Hyperextension von $5^{\circ}$ zeigen. Frauen wiesen in einer Studie von Amis u. Miller eine um $5-8^{\circ}$ größere Hyperextension im Vergleich zu Männern auf [10].

Eine weitere wichtige Rolle für eine regelrechte Funktionsfähigkeit des Ellenbogens in Flexion und Extension

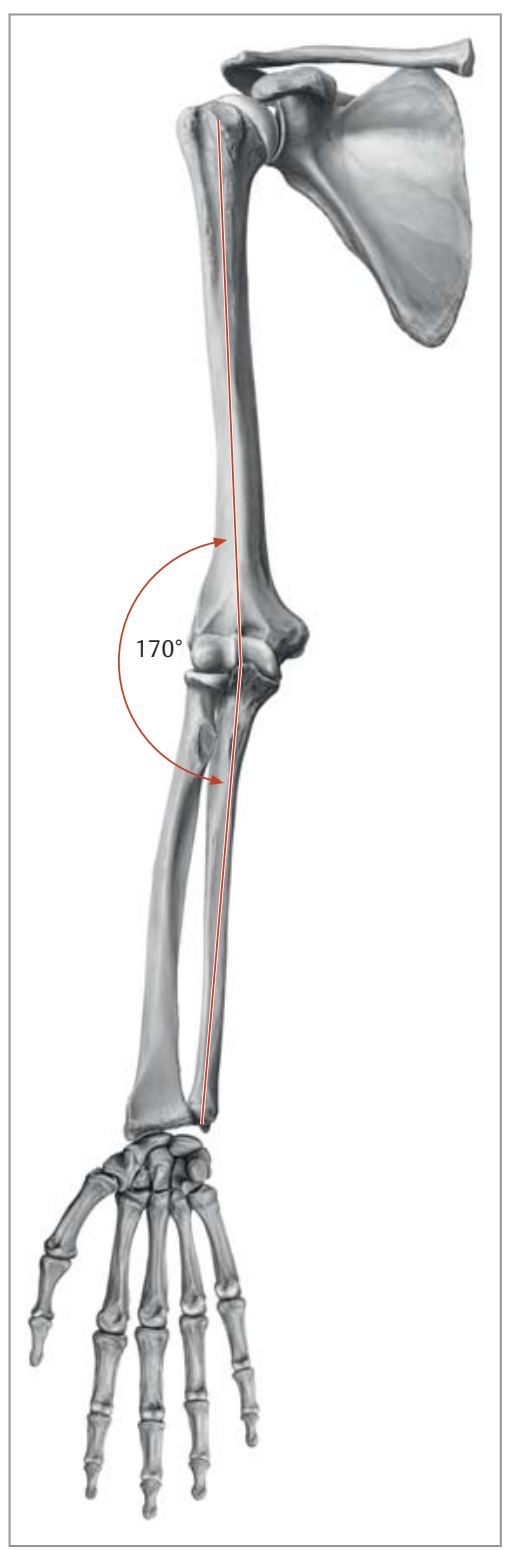

Abb. 9 -

Physiologische Valgusstellung im Ellenbogengelenk [3].

spielt der Valguswinkel („Tragewinkel“, engl.: „carrying angle“), der sich durch Schneiden der Längsachsen von Humerus und Ulna bei voll extendiertem Ellenbogengelenk und vollständig supiniertem Unterarm ergibt. Er beträgt $10-15^{\circ}$ bei Extension und ist im Mittel bei Frauen um 4-5 ${ }^{\circ}$ größer als bei Männern [9]. Der Winkel ergibt sich durch die distale Extension der Trochlea, die eine valgische Gelenkausrichtung zur Folge hat (Abb. 9).

In Flexion verändert sich der Winkel und erreicht in $120^{\circ}$ Beugung eine $6^{\circ}$-Varusstellung. Diese Veränderung entsteht durch die asymmetrische Form im Gelenk und die folglich schräg angeordnete Drehachse des humeroulnaren Gelenks. 


\section{Prinzipien}

\section{Chirurgische Relevanz}

Eine Zu- oder Abnahme über das genannte physiologische Maß hinaus kann mit einer zunehmenden Instabilität einhergehen [8].

\section{Pronation und Supination}

Als zweite Bewegungsrichtung erlaubt das Ellenbogengelenk eine Pronations-Supinations-Bewegung. Hierbei rotiert der Unterarm um seine Rotationsachse, die durch das Zentrum des Radiuskopfes und den Mittelpunkt des distalen Radioulnargelenks verläuft.

Die Umwendbewegung erfolgt aus dem humeroradialen Gelenk und dem proximalen Radioulnargelenk (PRUG).

Das Bewegungsausmaß beträgt physiologischerweise ca. $75 / 0 / 85^{\circ}$, wobei verschiedene Arbeiten zum Bewegungsausmaß voneinander stark abweichende Ergebnisse zeigen, die möglicherweise auf die hohe Individualität des proximalen Drehzentrums bei Differenzen in der geometrischen Form des Radiuskopfes zurückzuführen sind.

\section{Bandapparat}

Neben den Knochenstrukturen des Ellenbogengelenks sind die Kollateralbänder, die Verstärkungen der Gelenkkapsel darstellen, von Bedeutung (Abb. 10).

\section{Mediales Kollateralband}

Das mediale Kollateralband (MCL) besteht aus einem anterioren (A-MCL) und einem posterioren Bündel (P-MCL), die in ihren distalen Abschnitten durch das Lig. transversum verbunden sind.

\section{Anteriores Bündel des medialen Kollateralbandes}

(A-MCL). Das A-MCL entspringt am anteroinferioren Anteil des medialen Epikondylus und zieht zum Tuberculum subliminus am medialen Processus coronoideus.

Das A-MCL zeigt sich als das stabilste Ligament am
Ellenbogen mit einer Grenzlast von $261 \pm 71 \mathrm{~N}$.

Das A-MCL kann weiter in anteriore und posteriore Fasern unterteilt werden, wobei die anterioren Fasern vornehmlich in Streckung und die posterioren Fasern in Beugung gespannt werden [2].

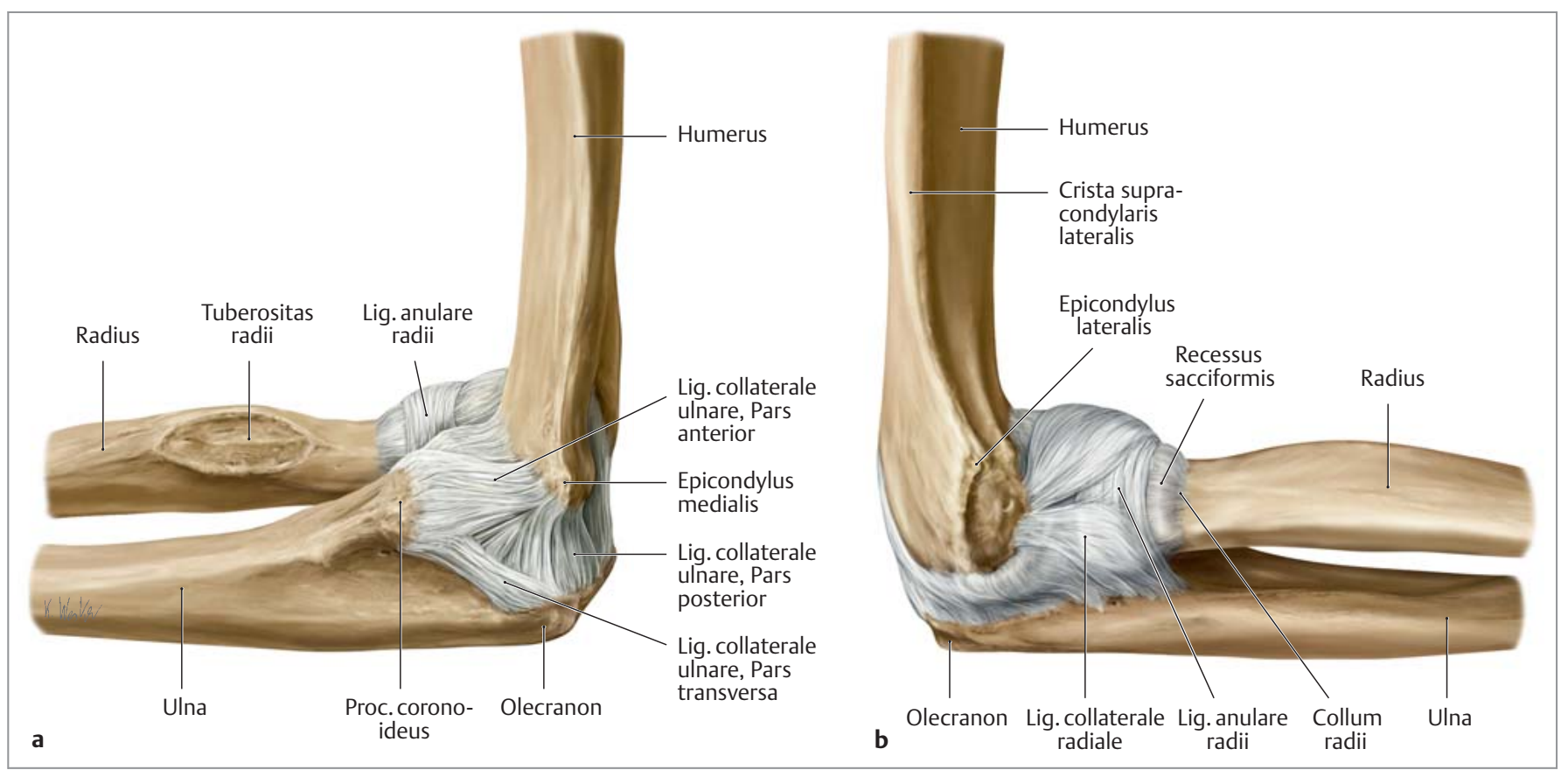

Abb. 10 - Kollateralbandapparat des Ellenbogens [3]. a Ansicht von medial. b Ansicht von lateral. 
Posteriores Bündel des medialen Kollateralbandes (P-MCL). Das P-MCL wiederum zieht vom anteroinferioren Epikondylus zum mittleren Abschnitt der Incisura semilunaris der Ulna. In Flexion ist es gespannt und in Extension locker. Es zeigte in Studien eine Belastbarkeit von $159 \pm 40 \mathrm{~N}$ [2].

In seiner Gesamtheit ist das MCL der entscheidende Stabilisator gegen Valgusbelastungen und wird in dieser Funktion vom Radiuskopf (Kompression am Capitulum) und dem ventralen Anteil der Gelenkkapsel unterstützt.

Das MCL ist der entscheidende Stabilisator gegen Valgusbelastungen.
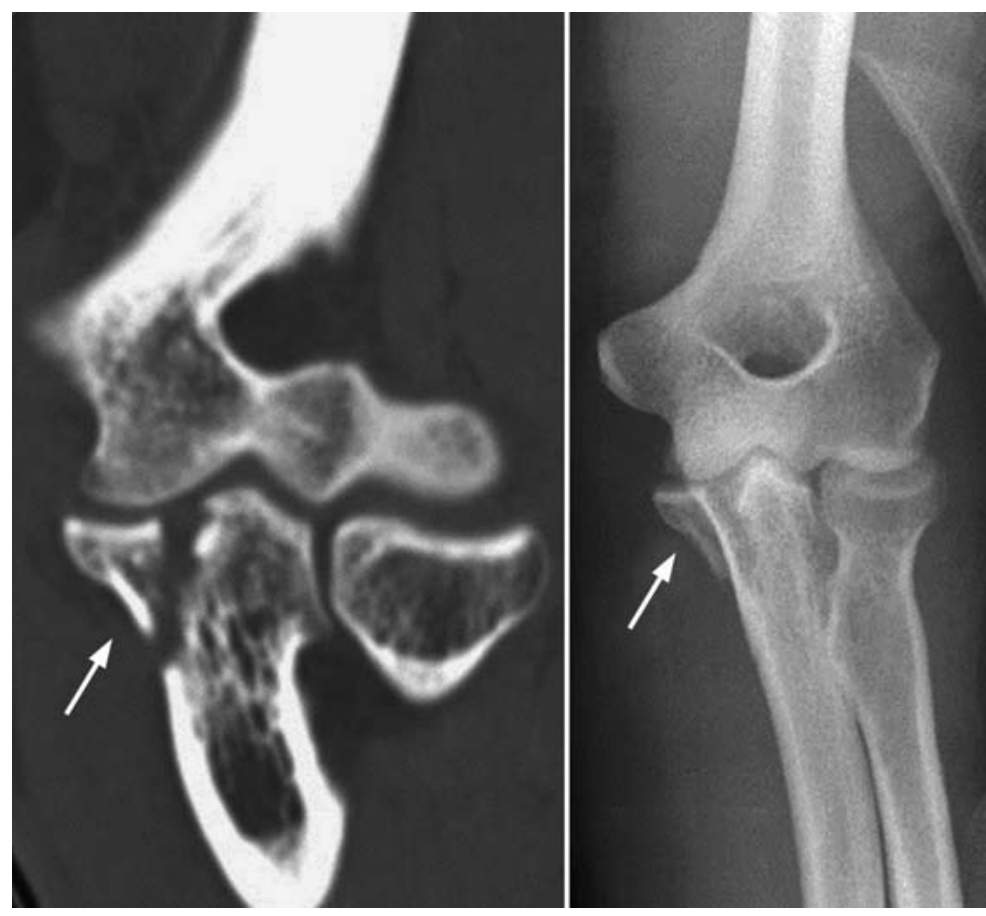

Abb. 11 - Die Fraktur des Tuberculum subliminus geht mit einer medialen Instabilität einher (Pfeile markieren das Frakturelement).

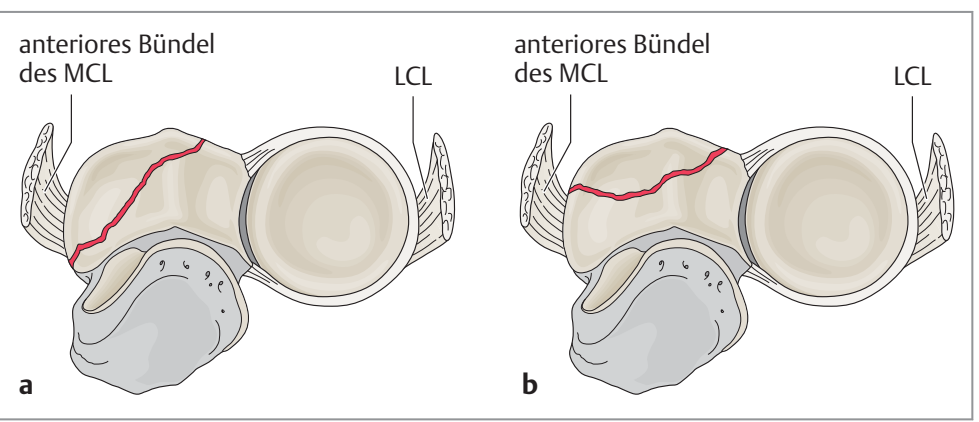

Abb. 12 - Abhängigkeit der Gelenkstabilität vom Frakturverlauf am Processus coronoideus [1]. a Instabile Verletzung bei Fraktur des Tuberculum subliminus. b Stabile Verletzung bei intaktem Tuberculum subliminus.

\section{Prinzipien}

\section{Chirurgische Relevanz}

Bleibt das Tuberculum subliminus (mit der Insertion des medialen Bandes) bei Frakturen des Processus coronoideus in der Frontalebene intakt, resultiert nicht zwangsläufig eine Valgusinstabilität. Verläuft die Frakturlinie mehr sagittal mit Fraktur des Tuberculum subliminus, geht dies meist mit einer relevanten Instabilität einher. Für die Differenzierung empfiehlt sich die exakte Beurteilung des Processus coronoideus und des Tuberculum subliminus im Röntgenbild bzw. der Computertomografie (Abb. 11 u. Abb. 12).

Diese Funktion kann beispielsweise bei $90^{\circ}$-Flexion des Ellenbogengelenks und innen- oder außenrotierter Schulter demonstriert werden. Die größte Belastung kann bei Innenrotation der Schulter und hohem Druck der Handfläche gegen ein Widerlager zentral vor dem Oberkörper bzw. bei einer kraftvollen Wurfbewegung erzeugt werden.

Junge Erwachsene erreichen bei Innenrotation des Humerus ein Drehmoment von bis zu 65 Nm, wodurch am Ellenbogen ein gleich großes Drehmoment mit entgegengesetztem Vorzeichen entsteht [10]. Diese Momente müssen durch das MCL, den Radiuskopf und den ventralen Anteil der Gelenkkapsel aufgefangen werden, was dem ca. 6-Fachen der äußeren Kraft am MCL und dem etwa 8-Fachen der äußeren Kraft am Radiuskopf entspricht.

Zeigt sich der Radiuskopf verletzt oder in seiner Form verändert, muss dieses Drehmoment durch Zug am MCL und Druck auf der lateralen Facette des Processus coronoideus aufgenommen werden. Hierdurch entstehen Kräfte, die zu einem erhöhten Valgusstress am Ellenbogen und zu sekundären Schäden führen können [2].

\section{Laterales Kollateralband}

Das laterale Kollateralband (LCL) setzt sich zusammen aus

- dem radialen Seitenband (RCL),

- dem Lig. anulare sowie

- dem lateralen ulnaren Seitenband (LUCL).

Es erreicht Grenzlasten von insgesamt ca. $233 \pm 16 \mathrm{~N}$ bei einer relativ niedrigen Steifigkeit [2]. 
Radiales Kollateralband (RCL). Das RCL entspringt am Epicondylus lateralis und strahlt in das Lig. anulare ein, sodass Drehbewegungen des Radius ungehindert durchgeführt werden können. Das Lig. anulare zieht ventral von der Incisura trochlearis ringförmig um die Circumferentia articularis des Radiuskopfes und setzt dorsal der Incisura trochlearis wieder an.

Akzessorisches laterales Kollateralband (ALCL). Das akzessorische laterale Kollateralband (ALCL) entspringt als Faseranteil des Lig. anulare und zieht ebenfalls zum Tuberculum der Crista m. supinatoris ulnae.

Laterales ulnares Kollateralband (LUCL). Das LUCL ist inkonstant ausgeprägt und entspringt zumeist am lateralen Epikondylus in enger Beziehung zum M. supinator, M. anconeus und dem gemeinsamen Ansatz der Extensoren (M. extensor carpi radialis longus et brevis, M. extensor digitorum und M. extensor carpi unaris), von wo aus es dorsal des radialen Kollateralbandes zur Ulna zieht und dort nach Verbindung mit Fasern des Lig. anulare breitbasig inseriert.

Das LCL schützt das Ellenbogengelenk gegenüber Varusstress und ermöglicht die Stabilisierung des ulnohumeralen und des radiokapitellaren Gelenkabschnittes, wenn der Unterarm in Supination belastet wird. Eine Läsion des LCL kann somit eine posterolaterale Rotationsinstabilität (PLRI) zur Folge haben, wodurch der Radiuskopf nach dorsal aus dem Radiohumeralgelenk luxiert. Eine weitere Außenrotation des Unterarms führt dazu, dass die Ulna aus dem Ulnohumeralgelenk nach dorsal herausdreht, wodurch zunächst der Rest des LCL zusammen mit der ventralen und posterioren Kapsel rupturiert, bevor das posteriore und gegebenenfalls auch das anteriore Bündel des MCL reißt.

Das LCL schützt das Ellenbogengelenk gegenüber Varusstress. Eine Läsion kann eine posterolaterale Rotationsinstabilität zur Folge haben.

\section{Prinzipien}

\section{Chirurgische Relevanz}

Der Ellenbogen ist im Alltag durch repetitive Abduktionsbewegungen hauptsächlich Varusstress ausgesetzt, wodurch es zu einer akuten oder chronischen Insuffizienz des lateralen Kollateralbandapparats kommen kann. Aus diesem Grund wird eine PLRI häufiger gesehen als eine mediale Instabilität.

\section{Periphere Nerven}

Die nervale Versorgung der auf den Ellenbogen wirkenden Muskulatur erfolgt durch 4 Nerven. Die Flexorengruppe wird vom $\mathbf{N}$. musculocutaneus innerviert, der durch die Teilinnervation des M. brachialis durch den N. radialis unterstützt wird. Bei der chirurgischen Präparation muss auf den $\mathbf{N}$. cutaneus antebrachii lateralis als Endast des N. musculocutaneus geachtet werden, der zwischen M. biceps brachii und M. brachialis lateralseitig nach distal verläuft.

Eine Läsion des N. musculocutaneus hebt nicht die komplette Flexion des Ellenbogens auf.

Die Extension des Ellenbogens erfolgt hauptsächlich über die posteriore Oberarmmuskulatur, die vom $\mathrm{N}$. radialis innerviert wird, der zwischen den Mm. brachialis und brachioradialis in die Ellenbeuge tritt und sich spätestens vor dem Radiuskopf in einen R. superficialis und einen R. profundus teilt.

\section{Prinzipien}

\section{Chirurgische Relevanz}

Da sich der R. profundus bei Supination um den Radiushals schlingt und somit nach proximal verlagert wird, kann durch Pronation eine größere Distanz zur Gelenkfläche erreicht werden, was bei chirurgischen Eingriffen die sogenannte Safe-Zone vergrößert.

Der N. radialis innerviert des Weiteren die supinatorisch wirksamen Muskeln, wohingegen die Pronatorgruppe vom N. medianus innerviert wird.

Als vierter wichtiger Nerv des Unterarms ist der N. ulnaris zu nennen, der nach Durchdringen des Septum intermusculare brachii mediale den medialen Kopf des M. triceps brachii überquert und zum Sulcus nervi ulnaris zwischen dem Epicondylus medialis und dem Olekranon läuft (Abb. 13). Danach erreicht er zwischen den beiden Muskelköpfen des M. flexor carpi ulnaris den Unterarm, wo er an der Ulnarseite auf dem M. flexor digitorum profundus weiter nach distal verläuft. 


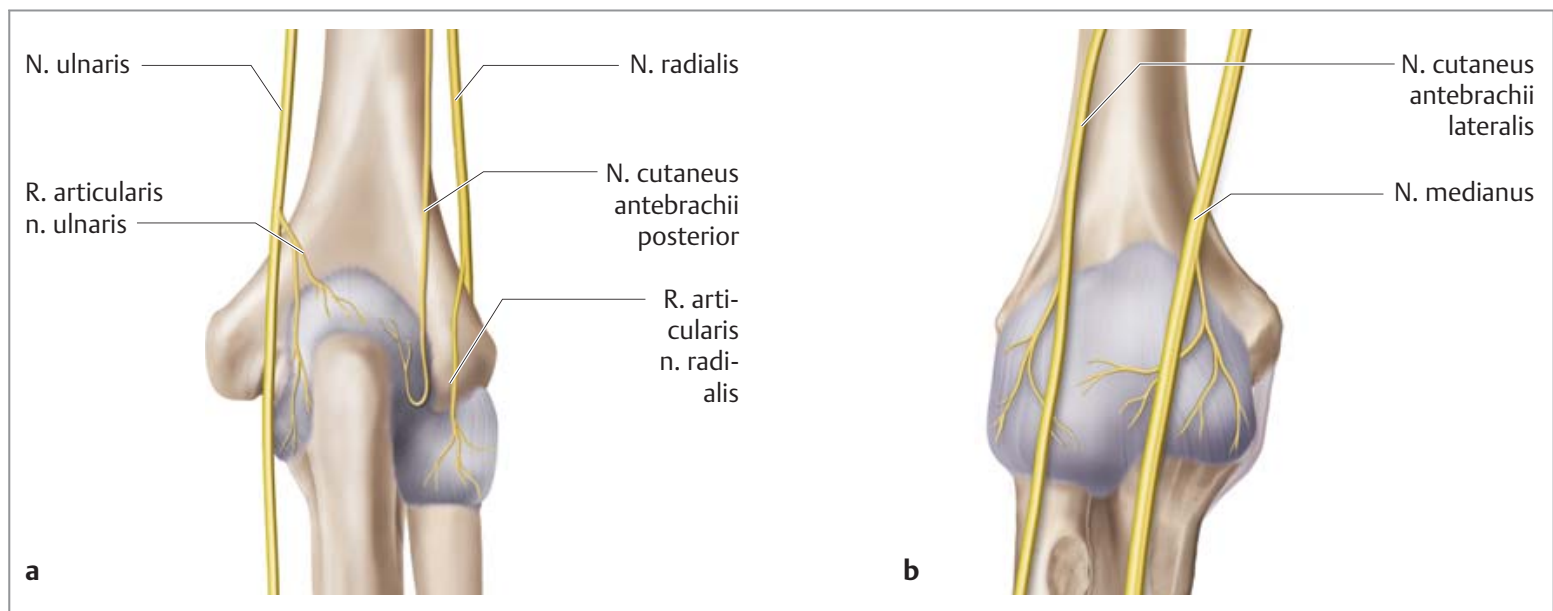

Abb. 13 - Nerven am Ellenbogengelenk. Innervation der A. cubiti [4]. a Ansicht von dorsal. b Ansicht von ventral.

\section{Muskulatur und Muskelkräfte}

Die Ellenbogenbewegungen sind neben dem Resultat der Gewichts- und Trägheitskräfte des Unterarms und der Hand insbesondere das Ergebnis der Kräfte der das Gelenk umgebenden Muskeln (Abb. 14). Diese lassen sich nach Funktion einteilen in die Gruppen

- Flexoren/Extensoren und

- Supination/Pronation.

Wichtig ist hierbei, dass jede Bewegung des Ellenbogengelenks das Resultat der Kraftwirkung von mehr als einem Muskel ist.

\section{Flexion im Ellenbogen}

Die Flexoren des Ellenbogengelenks bestehen aus der ventralen Oberarmmuskulatur und der radialen Unterarmmuskulatur. Die ventrale Oberarmmuskulatur besteht aus dem M. biceps brachii und dem M. brachialis.

M. biceps brachii. Der M. biceps brachii, der doppelköpfig mit seinem Caput longum am Tuberculum supraglenoidale und mit seinem Caput breve am Processus coracoideus entspringt und an einem gemeinsamen Ansatz an der Tuberositas radii einstrahlend in die Aponeurosis bicipitalis ansetzt, ist bei Flexion des Ellenbogengelenks nur bei supinierter oder neutraler Lage des Unterarms aktiv.

M. brachialis. Der M. brachialis hat einen oberflächigen Kopf mit dem Ursprung am anterolateralen Anteil des distalen Humerus bzw. beiden Septa intermuscularia; dieser oberflächige Kopf liegt proximal zum kleineren tiefen Kopf und gibt den größten Anteil der Muskelfasern ab, die an der Tuberositas ulnae ansetzen. Er ist, unabhängig von der Stellung, der primäre Flexor des Ellenbogens. Diese Funktion wird vor allem durch die oberflächigen Faseranteile erfüllt, wohingegen die tiefen Anteile bei der Startbewegung der Flexion aktiv werden.

Der oberflächige größere Kopf des M. brachialis ist der wichtigste Flexor im Ellenbogengelenk.

Als weitere wichtige Funktion gibt der M. brachialis Muskelfasern in die ventrale Gelenkkapsel ab, die bei Flexion ein Einklemmen der Kapsel verhindern. Nachteil seiner hohen Querschnittsfläche im Bereich der Kapsel ist die Gefahr für Verletzungen bei Ellenbogenluxationen.

\section{Prinzipien}

\section{Chirurgische Relevanz}

Aufgrund seiner großen Querschnittsfläche ist der M. brachialis anfällig für heterotope Ossifikationen postoperativ und Begleitläsionen im Rahmen von Ellenbogenluxationen [9].

M. brachioradialis. Als dritter Muskel der Flexorengruppe hat der M. brachioradialis seinen Ursprung an der Margo lateralis des Humerus, mit seinem Ansatz am distalen lateralen Aspekt des Radius nahe dem radialen Styloid. Bei schnellen Beugebewegungen des Unterarms oder beim Heben einer Last bei langsamer Flexion wird seine Hauptkraft entfaltet. 


\section{Biomechanik des Ellenbogengelenks}

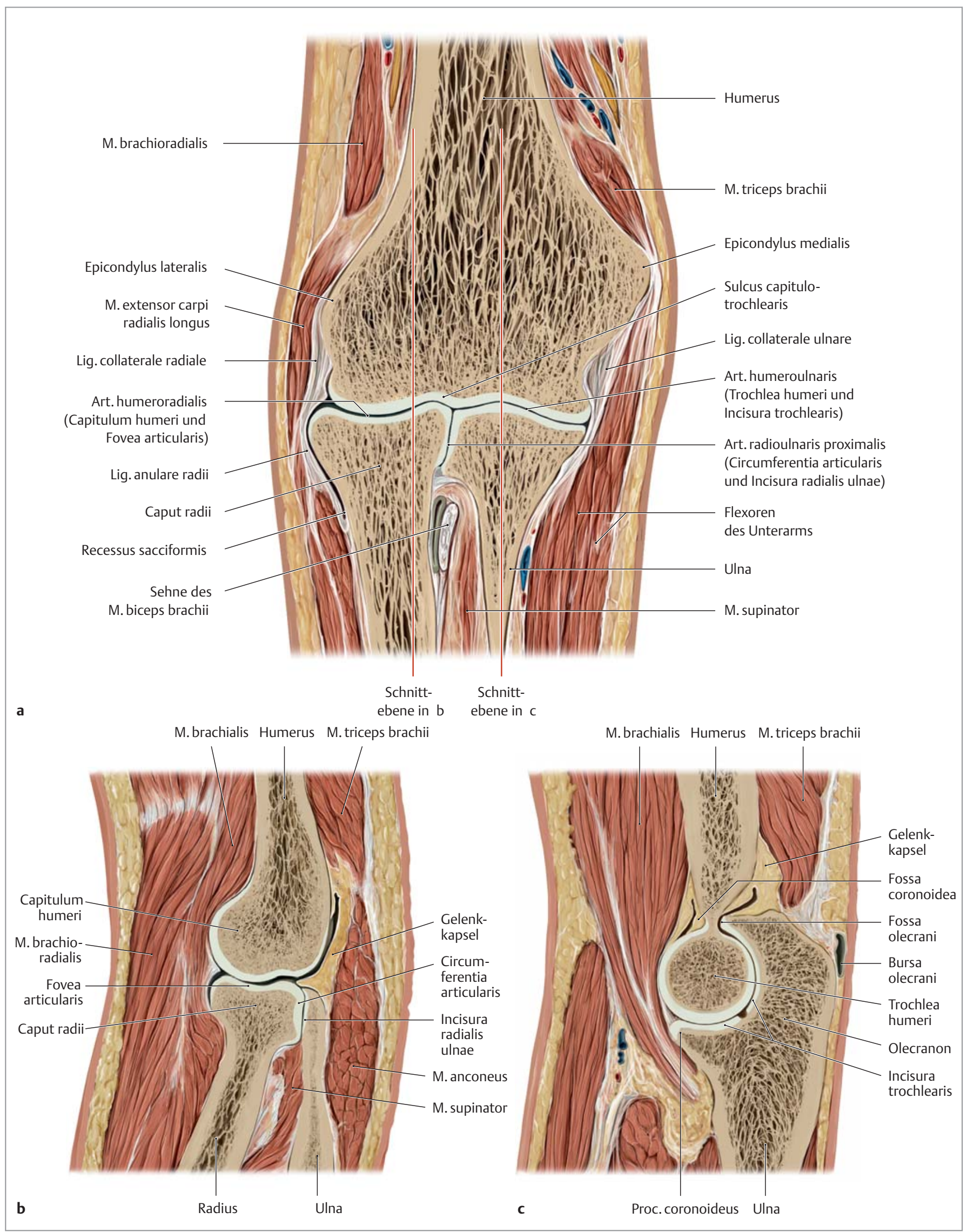

Abb. 14 - Muskeln des rechten Unterarms und des Ellenbogens [3]. a Frontalschnitt in der Ansicht von ventral. b Sagittalschnitt durch das Humeroradial- und das Radioulnargelenk in der Ansicht von medial. c Sagittalschnitt durch das Humeroulnargelenk in der Ansicht von medial. 
M. extensor carpi radialis. Als vierter Muskel zeigt der M. extensor carpi radialis ebenfalls ein Beugekraftpotenzial am Ellenbogen. Er entspringt an der Crista supracondylaris lateralis und inseriert dorsal an der Basis des Os metacarpale II. Neben seiner Funktion als Flexor im Ellenbogen streckt er typischerweise im Handgelenk.

Studien zur Kraftentfaltung zeigten eine optimale Muskellänge mit maximaler isometrischer Beugekraft bei einem Flexionswinkel von etwa $65^{\circ}$, wobei die maximale Beugekraft in Supination oder Neutralstellung des Unterarms generiert wird. Bei Pronation des Unterarms reduziert sich die maximale Flexionskraft um ca. $22 \%$. Die Reaktionskräfte in Flexion finden in distaler und posteriorer Richtung statt. Daraus ergibt sich ein Krafteintrag an der Basis des Koronoids und an der posterioren Lippe des Radiuskopfes, welcher jedoch abhängig von der Ellenbogenflexionsstellung ist.

\section{Prinzipien}

\section{Chirurgische Relevanz}

Der Krafteintrag an der Basis des Koronoids und an der posterioren Lippe des Radiuskopfes ist zum Verständnis von Versagensmechanismen, besonders von Radiuskopfprothesen, aber auch dem verfrühten Auftreten von arthrotischen Veränderungen wichtig.

\section{Extension}

M. triceps brachii. Der primäre Ellenbogenstrecker ist der M. triceps brachii. Er hat das größte Arbeits- und damit Kraftpotenzial für die Ellenbogenextension. Das Caput longum des Muskels entspringt am inferioren Rand des Glenoids der Skapula und zieht zwischen M. teres minor und major nach distal, wobei es diesen als Hypomochlion nutzt und auf diese Weise seinen Hebelarm verbessern kann. Hierdurch kann es als primärer Strecker des Ellenbogengelenks gesehen werden [13].

Der mediale und laterale Kopf sind am posterioren $\mathrm{Hu}-$ merus fixiert. Alle 3 Köpfe setzen mit einer gemeinsamen Sehne am Olekranon an, wobei sich beim Überspannen des Gelenks Muskelfasern abspalten und in die dorsale Kapsel einstrahlen. Auf diese Weise kann in Extension eine Einklemmung der Kapsel vermieden werden [11].
M. anconeus. Als zweiter Muskel zeigt sich der M. anconeus, der aus der lateralen Fortsetzung des Caput mediale hervorgeht, vor allem zu Beginn der Extension aktiv. Hauptfunktion des Muskels scheint indes die Stabilisierung des Ellenbogens bei Gesamtbewegungen der oberen Extremität zu sein, insbesondere zum Balancieren von externem Varusstress und posterolateralen Rotationskräften [11].

Das höchste Extensionsmoment von etwa $75 \mathrm{Nm}$ tritt bei $60^{\circ}$ Ellenbogenbeugung auf und ist als Resultat im Vergleich zum Flexionsmoment deutlich erhöht, was durch die verschiedenen Hebel und Kraftarme zu begründen ist, wobei die Positionierung des Unterarms hierbei zu vernachlässigen zu sein scheint. Auch die Gelenkkraft zeigt sich bei Extension um das 3-Fache gegenüber der Flexion erhöht. Aufgrund der kleinen Kraftarme der Extensionsmuskeln kann die resultierende Gelenkkraft mit Hauptwirkung am distalen Aspekt der Trochlea das bis zu 20-Fache der an der Hand wirkenden externen Lasten ausmachen [12].

\section{Umwendbewegungen}

Je nach Stellung des Unterarms können die Umwendbewegungen (Pronation/Supination) sowohl aus einer Kraftentfaltung der Flexoren als auch der Extensoren erfolgen. Zum Verständnis und der Ableitung der Zugrichtung des Muskels muss die Kreuzung der Pro- und Supinationsachse (Linie zwischen Caput radii und Caput ulnae) berücksichtigt werden.

Das größte Drehmoment ist für beide Bewegungen Pronation und Supination - bei einem $90^{\circ}$ flektierten Ellenbogen möglich. Das Pronationsmoment ist jedoch aufgrund der Dehnung der Pronatoren bei Supination des Unterarms deutlich größer (in $60^{\circ}$ beispielsweise $10 \mathrm{Nm}$ in Pronation und $2 \mathrm{Nm}$ in Supination).

\section{- Pronation}

Die M. pronator quadratus und M. pronator teres sind für die Pronation des Unterarms verantwortlich.

M. pronator teres. Der M. pronator teres, der am Epicondylus mediale und am Processus coronoideus entspringt, zieht zum lateralen Aspekt des supinierten Radius und inseriert etwa in der Mitte des Radiusschaftes. Eine Hypertrophie kann zum Pronator-teres-Syndrom (Kompression des N. medianus) führen. 
Der M. pronator teres ist der primäre Pronator des Unterarms unabhängig von der Lage des Unterarms oder der Flexionsstellung. Dies überrascht, da seine Muskellänge von der Flexion im Ellenbogengelenk abhängt. Biomechanische Studien zeigten außerdem eine geringe Beugefähigkeit dieses Muskels auf.

M. pronator quadratus. Der M. pronator quadratus wird besonders bei schneller und kraftvoller Pronation vermehrt beansprucht. Er hat seinen Ursprung am volaren Rand der distalen Ulna und inseriert am distalen und lateralen Aspekt des supinierten Radius.

\section{- Supination}

Die beiden Muskeln, die sich für die Supination des Unterarms verantwortlich zeigen, sind der M. supinator und der M. biceps brachii.

M. biceps brachii. Sein Verlauf wurde bereits oben beschrieben.

M. supinator. Der M. supinator zieht breitbasig proximal und distal vom lateralen Epikondylus des Humerus, dem LCL, dem Lig. anulare und von der Crista musculi supinatoris zum anterioren Aspekt des supinierten proximalen Radius. Die Supinationsfunktion des M. supinator ist zwar schwächer als die des M. biceps brachii, jedoch nicht von der Beugestellung des Ellenbogens abhängig.

\section{Blutversorgung}

Die arterielle Blutversorgung des distalen Humerus und des proximalen Unterarms erfolgt über ein Netz (Rete articulare cubiti) von rückläufig aufsteigenden Ästen der A. radialis und A. ulnaris (A. recurrens radialis et ulnaris), die untereinander und mit Kollateralarterien der A. brachialis anastomosieren. Durch diese anatomische Besonderheit ergibt sich eine Art „Wasserscheide“ im zentralen Bereich des distalen Humerus, der somit auf Zuflüsse von der kranial auslaufenden zentralen Arterie und der von medial und lateral über die Kondylen eintretenden Gefäße angewiesen ist (Abb. 15).

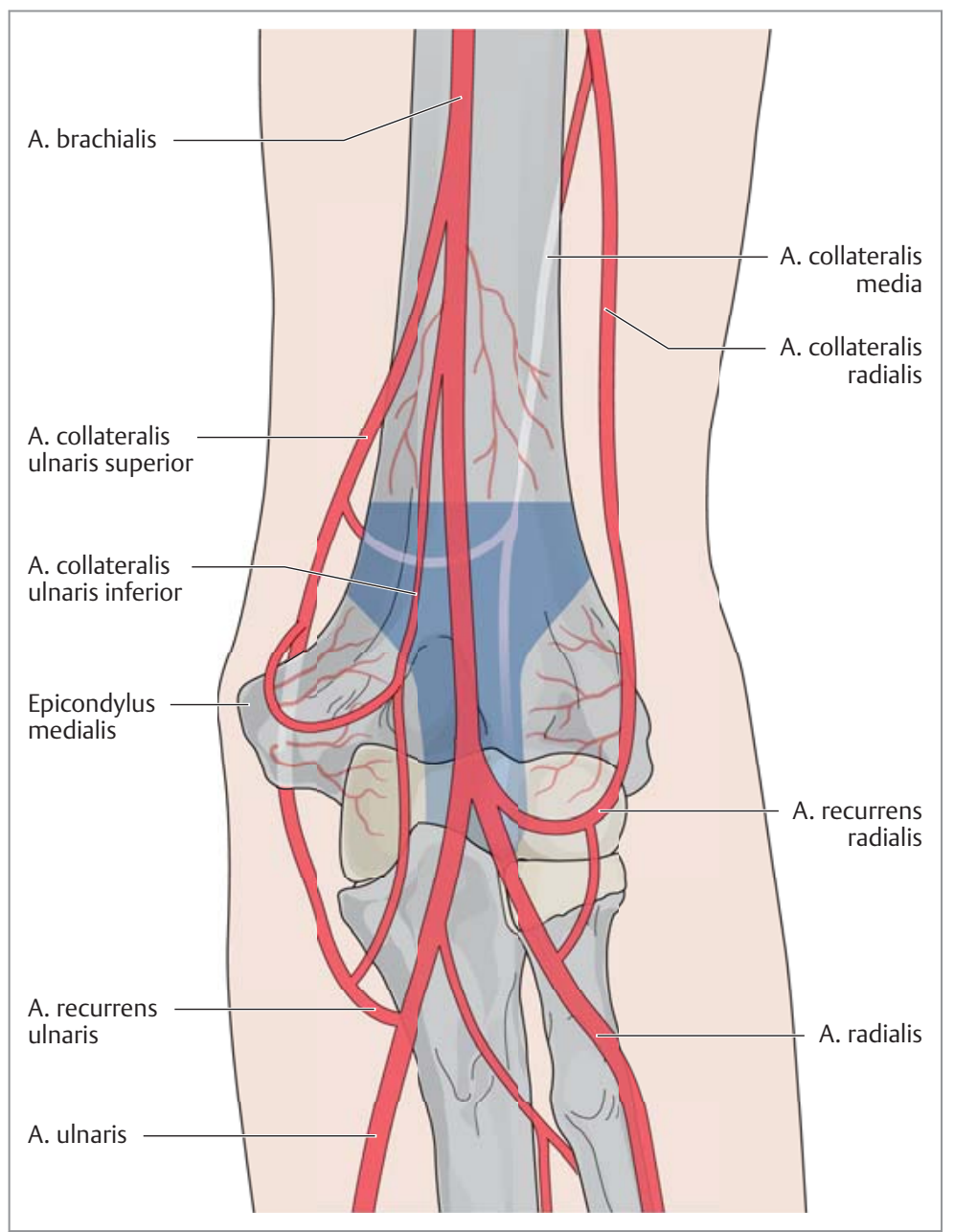

Abb. 15 - Die „Wasserscheide“ am distalen Humerus [1].

Im weiteren Verlauf zieht die A. radialis über den M. pronator und verläuft unter dem $M$. brachioradialis. Die A. ulnaris zieht unter dem M. pronator teres und dem M. flexor carpi ulnaris nach distal (Abb. 15) [1].

Interessenkonflikt: Die Autoren bestätigen, dass kein Interessenkonflikt vorliegt. 


\section{Grundlagen}

\section{Literatur}

1 Wegmann K, Burkhart KJ, Müller LP. Knöcherne Verletzungen des Ellenbogens. Orthop Unfallchir up2date 2012; 7: 339-364

2 Regan WD, Korinek SL, Morrey BF et al. Biomechanical study of ligaments around the elbow joint. Clin Orthop Relat Res 1991; 271: 170-199

3 Schünke M, Schulte E, Schumacher U. Prometheus. LernAtlas der Anatomie. Allgemeine Anatomie und Bewegungssystem. Illustrationen von M. Voll und K. Wesker. 3. Aufl. Stuttgart: Thieme; 2011

4 Hochschild J. Strukturen und Funktionen begreifen. Funktionelle Anatomie, Bd 1: Wirbelsäule und obere Extremität. 4. Aufl. Stuttgart: Thieme; 2014

5 Koebke J. Funktionelle Anatomie und Biomechanik des Ellenbogengelenkes. In: Stahl C, Koebke J et al., Hrsg. Klinische Arthrologie. Landsberg: Ecomed; 1998: 11

6 Schünke M, Schulte E, Schumacher U. Prometheus. LernAtlas der Anatomie. Allgemeine Anatomie und Bewegungssystem. Illustrationen von M. Voll und K. Wesker. 3. Aufl. Stuttgart: Thieme; 2011

7 Morrey BF, Askew LJ, An KN et al. A biomechanical study of normal elbow motion. J. Bone Joint Surg 1981: 63A: 872

8 Morrey BF. Complex instability of the elbow. Instr Course Lect 1998; 47: $157-164$
9 Morrey BF. Anatomy of the Elbow Joint. In: Morrey B, ed. The Elbow and its Disorders. Philadelphia: Saunders; 2000: 13-42

10 Amis AA. Biomechanics of the Elbow. In: Stanley D, Trail IA, eds. Operative Elbow Surgery. London: Churchill Livingstone Elsevier; 2012

11 Basmajian JV, Griffin WR jr. Function of anconeus muscle. An electromyographic study. J Bone Joint Surg Am 1972; 54: 1712 - 1714

12 Ishizuki M. Functional anatomy of the elbow joint and three-dimensional quantitative motion analysis of the elbow joint. Nihon Seikeigeka Gakkai Zasshi 1979; 53: 989 -996

13 Putz R, Milz S, Maier M et al. Funktionelle Morphologie des Ellenbogengelenks. Orthopäde 2003; 32: 684-690

14 Thomsen M, Loew M, Nägerl H. Kinematik und Biomechanik des Ellenbogengelenks. Orthopäde 2001; 30: 582 - 586

\section{Korrespondenzadresse}

Tim Leschinger

Schwerpunkt für Unfall-, Hand- und Ellenbogenchirurgie

Universitätsklinik zu Köln

Kerpener Straße 62

50937 Köln

E-Mail: tim.leschinger@uk-koeln.de 


\section{CME-Fragen}

Wie viel Grad umfasst der Umfang des Radiuskopfes, der als Bereich dienen sollte, in dem die osteosynthetische Versorgung ohne biomechanische Probleme erfolgen kann - die sogenannte Safe-Zone?

\section{CME.thieme.de}

\section{CME-Teilnahme}

- Viel Erfolg bei Ihrer CME-Teilnahme unter http://cme.thieme.de

- Bitte informieren Sie sich vorab online über die Gültigkeitsdauer.

- Sollten Sie Fragen zur Online-Teilnahme haben, unter http://cme.thieme.de/hilfe finden Sie eine ausführliche Anleitung.
Welche Stellung ist i.d.R. die aktuelle Ruhestellung des Ellenbogens?
A ca. $60^{\circ}$
B ca. $133^{\circ}$
C ca. $242^{\circ}$
D ca. $105^{\circ}$
E ca. $80^{\circ}$

Eine der folgenden Aussagen trifft auf den Unterarm als funktionelle Einheit nicht zu. Welche?
A $10-20^{\circ}$-Flexion
B $30-40^{\circ}$-Flexion
C $70-80^{\circ}$-Flexion
D $110-120^{\circ}$-Flexion
E $130-140^{\circ}$-Flexion

Wie groß ist das physiologische Füllungsvolumen der Gelenkkapsel des Ellenbogens?
A ca. $4-5 \mathrm{ml}$
B ca. $5-10 \mathrm{ml}$
C ca. $20-25 \mathrm{ml}$
D ca. $50-55 \mathrm{ml}$
E ca. $80-100 \mathrm{ml}$

Ulna ist wichtig.

Die Fasern der Membrana interossea verlaufen schräg abwärts nach medial (vom Radius zur Ulna).

Eine intakte Membrana interossea schützt vor Separation oder Migration von Radius und Ulna.

Es wird in anatomischen Studien von einer Festigkeitszunahme der Ulna und Festigkeitsabnahme des Radius von distal nach proximal berichtet.

Bei der Essex-Lopresti-Verletzung kommt es typischerweise zu einer Distalisierung des Radius.
Die Intaktheit des Tuberculum subliminus ist für die Stabilität des Ellenbogens wichtig, weil eine der im Folgenden genannten Strukturen an ihm inseriert. Welche?
A die Bizepssehne
B der M. supinator
C das anteriore Bündel des MCL
D das LUCL
E das Lig. anulare 


\section{Grundlagen}

Eine der folgenden Aussagen über die stabilisierende Wirkung des Radiuskopfes im Ellenbogengelenk trifft nicht zu. Welche?
A In Kombination mit dem medialen Kollateralband ist der Radiuskopf ein Valgusstabilisator.

B In Verbindung mit dem Processus coronoideus ist der Radiuskopf essenziell für die posterolaterale Rotationsstabilität.

C Der Radiuskopf ist ein wichtiger longitudinaler Kraftüberträger.

D Die Intaktheit des Humeroradialgelenks ist wichtig für die translatorische Stabilität des Ellenbogengelenks.

E Der Radiuskopf ist in Kombination mit dem MCL-Komplex der wichtigste Stabilisator gegen Varusstress.
Welcher Muskel ist der primäre Extensor des Ellenbogens?
A der M. brachialis
B der M. biceps brachii
C der M. triceps brachii
D der M. extensor carpi radialis
E der M. extensor carpi ulnaris

Eine der folgenden Aussagen über die peripheren Nerven am Ellenbogen trifft nicht zu. Welche?
A Eine Läsion des N. musculocutaneus hebt immer die komplette Flexion des Ellenbogen auf.

B Die Extension des Ellenbogens erfolgt hauptsächlich über die dorsale Oberarmmuskulatur, die vom $\mathrm{N}$. radialis innerviert wird.

C Bei der chirurgischen Präparation muss u.a. auf den N. cutaneus antebrachii lateralis geachtet werden.

D Die Flexorengruppe wird vom N. musculocutaneus innerviert, der durch die Teilinnervation des $\mathrm{M}$. brachialis durch den N. radialis unterstützt wird.

E Da sich der R. profundus bei Supination um den Radiushals schlingt und somit proximalisiert, kann durch Pronation eine größere Distanz zur Gelenkfläche erreicht werden, was bei chirurgischen Eingriffen die sogenannte Safe-Zone vergrößert.
Welches Band zählt nicht zum lateralen Kollateralbandkomplex (LCL)?
A das radiale Seitenband ( $R C L)$
B das Lig. anulare
C das laterale ulnare Seitenband (LUCL)
D das akzessorische laterale Kollateralband (ALCL)
E das Lig. transversum

Was ist das morphologische Korrelat des radiologischen Fat Pad Sign?
A eine weite Abhebung der Kapsel vom Knochen, die durch den intraartikulären Erguss verursacht wird

B eine extraartikuläre Einblutung

C ein Bone Bruise

D eine Teilläsion des M. triceps

E ein osteochondraler Flake 